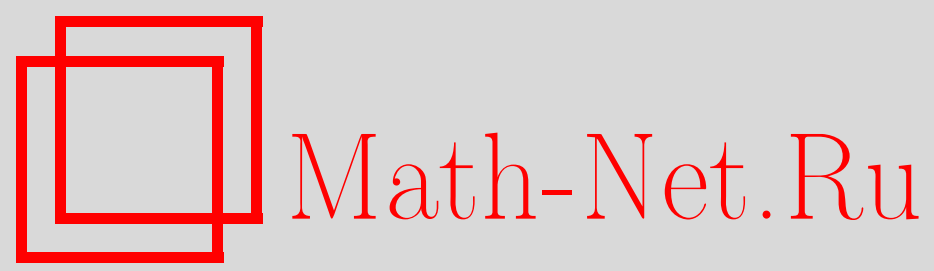

А. О. Шишанин, Матричные модели и паркетное приближение, Вестн. Сам. гос. техн. ун-та. Сер. Физ.-мат. науки, 2011, выпуск 1(), 172-178

DOI: https://doi.org/10.14498/vsgtu876

Использование Общероссийского математического портала Math-Net.Ru подразумевает, что вы прочитали и согласны с пользовательским соглашением http://www . mathnet.ru/rus/agreement

Параметры загрузки:

IP : 54.80 .97 .219

26 апреля 2023 г., 08:19:49 
УДК 530.14

\section{МАТРИЧНЫЕ МОДЕЛИ И ПАРКЕТНОЕ ПРИБЛИЖЕНИЕ}

\section{А.О. Шишанин}

Российский университет дружбы народов, 117198, Москва, ул. Миклухо-Маклая, 6.

E-mail: shishandr@rambler.ru

Рассматривается сравнение планарного и планарно-паркетного приближений в нульмерных эрмитовых матричных моделях. Обсуждается, как паркетное приближение воспроизводит результаты планарного подхода для матричной модели $\phi^{4}$, многоследовой модели, двуматричной модели и матричной модели Голдстоуна.

Ключевые слова: матричные модели, планарное приближение, уравнения Швингера-Дайсона, паркетнье уравнения.

Введение. Основным методом исследования в квантовой теории поля является теория возмущений, которая справедлива при малых константах связи. Паркетное приближение было предложено группой Ландау для исследования квантовой электродинамики и мезонной теории при больших импульсах. Данный подход производит суммирование большого класса диаграмм. При этом была записана замкнутая система уравнений на пропагаторы и вершинные функции, которая справедлива при любых значениях константы связи. Из анализа системы следовало, что квантовая электродинамика является асимптотически несвободной теорией, то есть она не работает при больших энергиях. В данной работе рассматривается применение планарного паркетного приближения к матричным моделям при больших размерах матриц.

Нульмерные матричные модели можно рассматривать как упрощённый вариант многомерных матричных теорий поля, для которых трудно построить $1 / N$-разложение (в данном случае $N$ - размер матрицы). Хорошо известно [1], что для калибровочной теории $U(N)$ в пределе больших $N$ и при условии $g^{2} N=$ const (где $g$ - константа связи теории Янга-Миллса) ведущий вклад дают планарные диаграммы (это диаграммы, которые можно нарисовать на плоскости). Брезан и другие [2] нашли вакуумную энергию и корреляторы для нульмерной эрмитовой матричной модели в главном (планарном) приближении по $1 / N$. Впервые планарное паркетное приближение было рассмотрено для тех же моделей Арефьевой и Зубаревым [3]. Было найдено замечательное согласие планарного и паркетного подходов для разных значений константы связи. Здесь будет рассмотрено обобщение этой процедуры на более сложные матричные теории поля: модель Голдстоуна, многоследовую модель, двуматричную модель.

1. Матричная модель $\phi^{\mathbf{4}}$. Действие матричной модели $\phi^{4}[2]$ имеет следующий вид:

$$
S(\Phi)=\frac{1}{2} \operatorname{Tr} \Phi^{2}+\frac{g}{4} \operatorname{Tr} \Phi^{4}
$$

Андрей Олегович Шишанин (к.ф.-м.н.), доцент, каф. экспериментальной физики. 
где $\Phi$-эрмитовая $N \times N$ матрица. Статсумма задаётся функциональным интегралом

$$
Z=\int d \Phi e^{-N S(\Phi)}
$$

Определим здесь планарную вакуумную энергию

$$
E^{0}(g)=-\lim _{N \rightarrow \infty} \frac{1}{N^{2}} \ln Z .
$$

В данном случае можно перейти к собственным значениям $\lambda_{i}$ матрицы $\Phi$ и написать для них уравнение следующего вида:

$$
\frac{1}{2}\left(\lambda_{i}+g \lambda_{i}^{3}\right)=\sum_{i \neq j} \frac{1}{\lambda_{i}-\lambda_{j}} .
$$

В планарном пределе, когда $N \rightarrow \infty, \lambda_{i}=\lambda(i / N), x=i / N$. Плотность распределения собственных значений матрицы определится как $u(\lambda)=d x / d \lambda$. Она удовлетворяет условию нормировки

$$
\int_{-2 a}^{2 a} d \lambda u(\lambda)=1
$$

В непрерывном пределе уравнение (1) при конечных $N$ переходит в интегральное уравнение на плотность $u(\lambda)$ :

$$
\frac{1}{2} \lambda+\frac{1}{2} g \lambda^{3}=\int_{-2 a}^{2 a} d \mu \frac{u(\mu)}{\lambda-\mu}, \quad|\lambda| \leqslant 2 a .
$$

Это уравнение имеет следующее решение [2] на отрезке $[-2 a, 2 a]$ :

$$
u_{A}=\frac{1}{\pi}\left(\frac{1}{2} g \lambda^{2}+\frac{1}{2}+g a^{2}\right) \sqrt{4 a^{2}-\lambda^{2}},
$$

где $a$ удовлетворяет условию $3 g a^{4}+a^{2}-1=0$.

Пропагатор, или двухточечная корреляционная функция, здесь определяется выражением

$$
D_{p l}=\int_{-2 a}^{2 a} d \lambda u(\lambda) \lambda^{2}=\frac{\left(4+a^{2}\right) a^{2}}{3} .
$$

Замечательным результатом статьи [2] является вычисление планарной вакуумной энергии:

$$
E^{0}(g)-E^{0}(0)=\frac{1}{24}\left(a^{2}-1\right)\left(9-a^{2}\right)-\frac{1}{2} \ln a^{2} .
$$

Посмотрим, как рассмотренная модель воспроизводится паркетным приближением [3]. Замкнутая система планарного уравнения Швингера-Дайсона на пропагатор и планарных паркетных уравнений в данном случае выглядит как

$$
D=1-2 g D^{2}-g D^{4} \Gamma_{4}, \quad \Gamma_{4}=-g+H+V,
$$




$$
H=-g D^{2} \Gamma_{4}+V D^{2} \Gamma_{4}, \quad V=-g D^{2} \Gamma_{4}+H D^{2} \Gamma_{4} .
$$

Здесь $D$ - пропагатор, $\Gamma_{4}$ - четырёхточечная вершина, $H$ и $V$-планарные паркетные интегральные ядра (эти обозначения используются и для других моделей). Отметим, что в этих уравнениях не учитывается ещё один канал, который принимается во внимание в скалярном случае. Если исключить из системы $\Gamma_{4}, H, V$, то для пропагатора можно записать следующее уравнение:

$$
g^{3} D^{6}+g^{2} D^{5}+5 g^{2} D^{4}+5 g D^{3}+(1-5 g) D^{2}-2 D+1=0 .
$$

Проверено,что при малых значениях $D_{p a r}$ совпадает с $D_{p l}$ до шестого порядка по константе связи. Более интересно сравнить для двух подходов предел сильной связи, когда константа связи $g \rightarrow \infty$. На $D_{p r}$ можно получить следующее асимптотическое поведение в этом режиме:

$$
D(g) \sim \frac{\alpha_{p a r}}{\sqrt{g}},
$$

где $\alpha_{\text {par }}-$ решение уравнения $\alpha_{\text {par }}^{6}+5 \alpha_{\text {par }}^{4}-5 \alpha_{\text {par }}^{2}+1=0$. Одним из корней этого уравнения является $\alpha_{p a r}=0,76948$. Можно показать, что планарный пропагатор удовлетворяет уравнению

$$
27 g^{2} D_{p l}^{2}+(1+18 g) D_{p l}-1-16 g=0 .
$$

Тогда асимптотическое поведение планарного пропагатора $D_{p l}$ запишется так:

$$
D(g)_{p l} \sim \frac{\alpha_{p l}}{\sqrt{g}}
$$

где берётся положительный корень

$$
\alpha_{p l}=4 \sqrt{3} / 9=0,7698 .
$$

Решение паркетного уравнения имеет фазовый переход при $g=g_{p r}$, $-0,0865<g_{p a r}<-0,0864$. В планарном подходе $g_{p l}=-1 / 12=-0,0833(3)$.

2. Многоследовая матричная модель. Многоследовая матричная модель была предложена в [4], и её действие выглядит так:

$$
S(\Phi)=\frac{1}{2} \operatorname{Tr} \Phi^{2}+\frac{g}{4} \operatorname{Tr} \Phi^{4}+\frac{h}{4}\left(\operatorname{Tr} \Phi^{2}\right)^{2} .
$$

Этот случай решается аналогично предыдущему. Можно получить плотность распределения собственных значений в форме

$$
u(\lambda)=\frac{1}{\pi}\left(\frac{1}{2}+2 h D+g a^{2}+\frac{1}{2} g \lambda^{2}\right) \sqrt{4 a^{2}-\lambda^{2}} .
$$

Здесь $a$ удовлетворяет уравнению

$$
g h a^{8}+(3 g+h) a^{4}+a^{2}-1=0,
$$


а пропагатор $D$ можно представить в виде

$$
D=\frac{4 g a^{6}+a^{4}}{1-4 h a^{4}}
$$

Вакуумная энергия определяется выражением

$$
E^{0}(g, h)-E^{0}(0,0)=\frac{1}{4}\left(a^{2}-1\right)+\left(\frac{3 g a^{4}}{2}+a^{2}-1\right) \frac{g a^{4}}{4}-\frac{1}{2} \ln a^{2} .
$$

Этот результат является обобщением формулы (2).

При малых константах связи $g$ и $h$ физические величины определятся как

$$
\begin{aligned}
& E^{0}(g, h)-E^{0}(0,0)=2 g+\frac{1}{4} h-18 g^{2}-\frac{1}{4} h^{2}-4 g h+288 g^{3}+h^{2}+\ldots, \\
& D=1-2 g-h+9 g^{2}+2 h^{2}+8 g h-54 g^{3}-66 g^{2} h-30 g h^{2}-5 h^{3}+\ldots .
\end{aligned}
$$

Член многоследового взаимодействия не меняет вид паркетных уравнений, а уравнение Швингера-Дайсона примет вид

$$
D=1-2 g D^{2}-g D^{4} \Gamma_{4}-h D^{2} .
$$

В [5] проверено, что при малых значениях констант связи $g$ планарный и планарно-паркетный пропагаторы совпадают вплоть до пятого порядка разложения. В паркетном подходе четырёхточечная вершина не зависит от константы связи $h$, поскольку она входит только в планарное уравнение Швингера-Дайсона. В планарном же случае при малых $g$ вершинная функция задаётся соотношением

$$
\Gamma_{4}=-g+2 g^{2}-14 g^{3}+114 g^{4}+64 g^{3} h+10 g^{2} h^{2}+\ldots .
$$

В табл. 1 приводится сравнение для двух подходов критических констант связи. Наибольшее согласие двух подходов для критической константы связи $g$ будет, когда $h=0$, а в противоположном случае происходит их расхождение.

Таблица 1

Значения критических констант связи $g$ и $h$

\begin{tabular}{c|ccccccccc}
\hline$h / 128$ & 0 & -1 & -2 & -3 & -4 & -5 & -6 & -7 & -8 \\
\hline$g_{p l}$ & $-0,0865$ & $-0,0762$ & $-0,0687$ & $-0,0608$ & $-0,0518$ & $-0,0413$ & $-0,029$ & $-0,0151$ & 0 \\
$g_{p a r}$ & $-0,0833$ & $-0,0787$ & $-0,0706$ & $-0,0618$ & $-0,0522$ & -0.0414 & $-0,029$ & $-0,0151$ & 0 \\
\hline
\end{tabular}

3. Двуматричная модель. Действие двуматричной модели предложили Ициксон и Зюбер [6] в виде

$$
S\left(M_{1}, M_{2}\right)=\frac{1}{2} \operatorname{Tr} M_{1}^{2}+\frac{g}{4} \operatorname{Tr} M_{1}^{4}+\frac{1}{2} \operatorname{Tr} M_{2}^{2}+\frac{g}{4} \operatorname{Tr} M_{2}^{4}-c \operatorname{Tr} M_{1} M_{2} .
$$

Для простоты можно взять приближённо те же самые паркетные уравнения, что и в первом случае. Планарные уравнения Швингера-Дайсона [8] здесь будут следующими:

$$
D=1-2 g D^{2}-g D^{4} \Gamma_{4}-g S^{4} D \Gamma_{4}+c S,
$$




$$
S=c D-2 g S D-g S D^{3} \Gamma_{4}-g S^{3} D \Gamma_{4},
$$

где $D=\left\langle\frac{1}{N} \operatorname{Tr} M_{i}^{2}\right\rangle, i=1,2 ; S=\left\langle\frac{1}{N} \operatorname{Tr} M_{1} M_{2}\right\rangle$. Однотипный по полям пропагатор совпал до второго порядка по константе связи с планарным аналогом:

$$
D_{p l}=D_{1}=D_{2}=\frac{1}{1-c^{2}}-2 \frac{1+c^{2}}{\left(1-c^{2}\right)^{3}} g+\frac{9+21 c^{2}+6 c^{4}}{\left(1-c^{2}\right)^{5}} g^{2}+\ldots .
$$

В непрерывном пределе, когда $N \rightarrow \infty$, можно получить планарный пропагатор, используя биортогональные полиномы и технику, предложенную Казаковым для вычисления вакуумной энергии [7]. Точное выражение для планарного пропагатора имеет вид

$$
D_{p l}=\frac{w^{2}}{g^{2}}\left[1+4 w-\frac{c^{2}(1+5 w)}{(1+3 w)^{3}}\right]
$$

где $w$ выражается через $g$ как

$$
g(w)=w+3 w^{2}+\frac{3 c^{2} w^{3}}{(1+3 w)^{3}}-\frac{c^{2} w}{1+3 w} .
$$

Значения критических значений константы связи $g$ двух подходов при разных значениях $c$ сравниваются в нижеприведенной табл. 2.

Таблица 2

Зависимость $\boldsymbol{g}(\boldsymbol{c})$ для двух подходов

\begin{tabular}{c|cccccccccc}
\hline$c$ & 0 & 0,1 & 0,2 & 0,3 & 0,4 & 0,5 & 0,6 & 0,7 & 0,8 & 0,9 \\
\hline $0,01 g_{p l}$ & $-8,33$ & $-8,11$ & $-7,44$ & $-6,34$ & $-4,99$ & $-3,64$ & $-2,42$ & $-1,4$ & $-0,64$ & $-0,16$ \\
$0,01 g_{\text {par }}$ & $-8,64$ & $-8,4$ & $-7,72$ & $-6,67$ & $-5,4$ & $-4,04$ & $-2,72$ & $-1,62$ & $-0,74$ & $-0,16$ \\
\hline
\end{tabular}

4. Матричная модель Голдстоуна. Рассмотрим модель Голдстоуна с действием

$$
S(\Phi)=-\frac{1}{2} \operatorname{Tr} \Phi^{2}+\frac{g}{4} \operatorname{Tr} \Phi^{4} .
$$

Помимо одноразрезного решения здесь при некотором значении константы связи $g(\xi)$ имеется решение на двух отрезках $[a, b] \cup[c, d]$ для $u(\lambda)[9]$. Это решение можно характеризовать специальным параметром

$$
\xi=\int_{a}^{b} u(\lambda) d \lambda
$$

Паркетное приближение для полностью несимметричного случая $\xi=1$ рассмотрено в статье [11]. Здесь мы обсудим симметричную фазу на двух отрезках $\xi=1 / 2$ с $g_{k r}=1 / 4$. Было показано $[9,10]$, что плотность распределения

$$
u(\lambda)=\frac{g|\lambda|}{2 \pi} \sqrt{\left(b^{2}-\lambda^{2}\right)\left(\lambda^{2}-a^{2}\right)} .
$$

При данном подходе вакуумная энергия и пропагатор имеют следующий вид:

$$
E_{\text {two-cut }}=-\frac{1}{4 g}+\frac{1}{4} \ln g-\frac{3}{8}-2 \ln 2, \quad D=1 / g .
$$


Четырёхточечная вершина в этом случае определяется выражением $\Gamma_{4}=$ $=-g^{2}-g^{3}$. В симметричном случае можно считать, что свободный пропагатор равен -1 . Здесь паркетные уравнения имеют такой же вид, как и в обычной модели, рассмотренной выше, а уравнение Швингера-Дайсона выглядит так:

$$
D=-1+2 g D^{2}+g D^{4} \Gamma_{4}
$$

Уравнение на пропагатор имеет вид

$$
g^{3} D^{6}-g^{2} D^{5}+\frac{5}{4} g^{2} D^{4}-5 g D^{3}+(1-5 g) D^{2}+2 D+1=0 .
$$

Численный расчёт, результаты которого приведены в табл. 3, показывает,что когда $g \rightarrow 0$, имеется корень уравнения (4), который ведёт себя как $1 / g$.

Таблица 3

Пропагатор и вершинная функция симметричного решения

\begin{tabular}{c|cccccc}
\hline$g$ & 0,25 & 0,1 & 0,05 & 0,01 & 0,001 & $10^{-4}$ \\
\hline$D^{\text {par }}$ & 5,6 & 12 & 22,3 & 102,6 & 1002,7 & 100027 \\
$D^{(p l)}$ & 4 & 10 & 20 & 100 & 1000 & $10^{4}$ \\
\hline$\Gamma_{4}^{\text {par }}$ & $-0,037$ & $-7,6 \cdot 10^{-3}$ & $-2,1 \cdot 10^{-3}$ & $-9,6 \cdot 10^{-4}$ & $-9,96 \cdot 10^{-6}$ & $-54,38$ \\
$\Gamma_{4}^{(p l)}$ & $-0,078 \cdot 10^{-4}$ & $-9,813 \cdot 10^{-3}$ & $-0,08786$ & $-0,6900$ & $-5,835$ & $-54,55$ \\
\hline
\end{tabular}

5. Заключение. В данной работе были представлены результаты сравнения двух подходов: планарного и планарно-паркетного для матричных моделей. Хорошо известно [3], что паркетное приближение достаточно точно воспроизводит планарное приближение для матричной модели $\phi^{4}$. Здесь же было проверено, что этот подход достаточно точно аппроксимирует планарное приближение для многоследовой, двуматричной и голдстоуновской моделей.

Работа выполнена в рамках ГК П 23-13 Федеральной целевой программы «Научные и научно-педагогические кадры инновационной России».

\section{БИБЛИОГРАФИЧЕСКИЙ СПИСОК}

1. Hooft G.'t. A planar diagram theory for strong interactions // Nucl. Phys. B., 1974. Vol. 72, no. 3. Pp. 461-473.

2. Brézin E., Itzykson C., Parisi G., Zuber J. B. Planar diagrams // Commun. Math. Phys., 1978. Vol. 59, no. 1. Pp. 35-51.

3. Aref'eva I. Ya., Zubarev A. P. Parquet approximation in large $N$ matrix theories // Phys. Lett. B., 1996. Vol. 386, no. 1-4. Pp. 258-268, arXiv: hep-th/9605005.

4. Das S.R., Dhar A., Sengupta A.M., Wadia S. R. New critical behavior in $d=0$ large-N matrix models // Mod. Phys. Lett. A., 1990. Vol.5, no. 13. Pp. 1041-1056.

5. Шишанин А. О. Матричная многоследовая модель в паркетном приближении // Изв. вузов. Физика, 2005. № 12. С. 65-69; англ. пер.: Shishanin A. O. Multitrace matrix model in the parquet approximation // Russian Physics Journal, 2005. Vol.48, no. 12. Pp. 12871293.

6. Itzykson C., Zuber J. B. The planar approximation. II// J. Math. Phys., 1980. Vol. 21, no. 3. Pp. 411-421.

7. Kazakov V. A. Ising model on a dynamical planar random lattice: exact solution // Phys. Lett. A., 1986. Vol. 119, no. 3. Pp. 140-144. 
8. Шишанин А. О. Решение паркетных уравнений для двуматричной модели // Изв. вузов. Физика, 2006. № 5. С. 92-95; англ. пер.: Shishanin A. O. A solution to the parquet equations for a two-matrix model// Russian Physics Journal, 2006. Vol. 49, no. 5. Pp. 559562.

9. Shimamune $Y$. On the phase structure of large $N$ matrix models and gauge models // Phys. Lett. B., 1982. Vol. 108, no. 6. Pp. 407-411.

10. Cicuta G.M., Molinari L., Montaldi E. Large $N$ phase transitions in low dimensions // Mod. Phys. Lett. A, 1986. Vol.1, no. 2. Pp. 125-129.

11. Shishanin A., Ziatdinov I. Parquet approximation for large- $N$ matrix Higgs model // JHEP, 2003. Vol. 2003, no. 07, 032. 12 pp., arXiv: hep-th/0303169.

Поступила в редакцию 20/XII/2010;

в окончательном варианте - 20/III/2011.

\section{MSC: 81T10}

\section{MATRIX MODELS AND PARQUET APPROXIMATION}

\section{A. O. Shishanin}

Peoples Friendship University of Russia,

6, Mikluho-Maklai st., Moscow, 117198, Russia.

E-mail: shishandr@rambler.ru

In this work we consider the comparison of planar and planar parquet approximations for zero-dimensional hermitian matrix models. We discuss how the parquet approach reproduces planar one for matrix model $\phi^{4}$, multi-trace model, two-matrix model and the Goldstone matrix model.

Key words: matrix models, planar approximation, Schwinger-Dyson equations, parquet equations.

Original article submitted 20/XII/2010; revision submitted 20/III/2011.

Andrei O. Shishanin (Ph. D. (Phys. \& Math.)), Associate Professor, Dept. of Experimental Physics. 